\title{
Hypersonic Simulations using Open-Source CFD and DSMC Solvers
}

\author{
V. Casseau ${ }^{1,2, a)}$, T. J. Scanlon ${ }^{1,2}$, B. John ${ }^{3}$, D. R. Emerson ${ }^{3}$ and R. E. Brown ${ }^{2}$ \\ ${ }^{1}$ James Weir Fluids Laboratory, Department of Mechanical and Aerospace Engineering, University of Strathclyde, \\ Glasgow, G1 1XJ, UK \\ ${ }^{2}$ Centre for Future Air-Space Transportation Technology, Department of Mechanical and Aerospace Engineering, \\ University of Strathclyde, Glasgow, G1 IXJ, UK \\ ${ }^{3}$ Scientific Computing Department, STFC Daresbury Laboratory, Warrington, WA4 4AD, UK
}

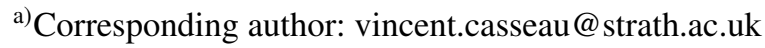

\begin{abstract}
.
Hypersonic hybrid hydrodynamic-molecular gas flow solvers are required to satisfy the two essential requirements of any high-speed reacting code, these being physical accuracy and computational efficiency. The James Weir Fluids Laboratory at the University of Strathclyde is currently developing an open-source hybrid code which will eventually reconcile the direct simulation Monte-Carlo method, making use of the OpenFOAM application called dsmcFoam, and the newly coded open-source twotemperature computational fluid dynamics solver named hy2Foam. In conjunction with employing the CVDV chemistry-vibration model in hy2Foam, novel use is made of the QK rates in a CFD solver. In this paper, further testing is performed, in particular with the CFD solver, to ensure its efficacy before considering more advanced test cases. The hy2Foam and dsmcFoam codes have shown to compare reasonably well, thus providing a useful basis for other codes to compare against.
\end{abstract}

\section{INTRODUCTION}

Hypersonic hybrid hydrodynamic-molecular gas flow solvers need to satisfy the two essential requirements of any high-speed reacting code, these being physical accuracy and computational efficiency. Available hybrid solvers were reviewed by Schwartzentruber and Boyd [1] and are essentially in-house codes, whilst the James Weir Fluids Laboratory at the University of Strathclyde is currently developing an open-source hybrid code [2].

The newly coded open-source two-temperature computational fluid dynamics (CFD) solver hy2Foam has been developed to tackle the highly complex flow physics of the hypersonic planetary atmospheric entry [3]. Implemented within the OpenFOAM framework [4], the code has the capability to model physical phenomena relative to the high-speed, chemically-reacting environment surrounding a spacecraft. The non-equilibrium conditions are treated by making the distinction between the trans-rotational and multiple vibrational-electronic energy pools. This allows the modelling of energy exchanges between the pools and the introduction a chemistry-vibration source term into the Navier-Stokes-Fourier (NSF) equations. hy2Foam has been extensively validated for a 5-species air using fundamental zero-dimensional benchmark cases.

The purpose of this work is to compare the results given by the hy2Foam and dsmcFoam solvers. The flowfield around a Mach 20 circular cylinder in the bottom range of the continuum-transition regime is investigated with emphasis being made on the importance of considering chemical reactions to correctly estimate the aerothermal loads.

\section{METHODOLOGY}

\section{Conventional computational fluid dynamics}

The computation of transient hypervelocity reacting flows in the continuum regime traditionally employs the nonequilibrium Navier-Stokes-Fourier equations. These are shown below in flux-divergence form using a Cartesian coor- 
dinate system for a mixture composed of $N_{s}$ species, including $N_{m}$ molecules [5]

$$
\frac{\partial \mathcal{U}}{\partial t}+\frac{\partial\left(\mathcal{F}_{i, i n v}-\mathcal{F}_{i, v i s}\right)}{\partial x_{i}}=\dot{\boldsymbol{W}}
$$

The vector of conserved quantities, $\mathcal{U}$, is defined as

$$
\mathcal{U}=\left(\rho, \rho_{s}, \rho u, \rho v, \rho w, E_{v e, m}, E\right)^{T} \quad s \in N_{s}, m \in N_{m}
$$

where $u, v$, and $w$ are the components of the velocity vector. $\rho$ is the mass density of the fluid and $\rho_{s}$ is the partial density of species $s$. The flux vectors are split into inviscid and viscous contributions and are written as follows

$$
\mathcal{F}_{i, i n v}=\left(\begin{array}{c}
\rho u_{i} \\
\rho_{s} u_{i} \\
\rho u_{i} u+\delta_{i 1} p \\
\rho u_{i} v+\delta_{i 2} p \\
\rho u_{i} w+\delta_{i 3} p \\
E_{v e, m} u_{i} \\
(E+p) u_{i}
\end{array}\right) \quad(3) \quad \mathcal{F}_{i, v i s}=\left(\begin{array}{c}
0 \\
-\mathcal{J}_{s, i} \\
\tau_{i 1} \\
\tau_{i 2} \\
\tau_{i 3} \\
-q_{v e, m, i}-e_{v e, m} \mathcal{J}_{m, i} \\
\tau_{i j} u^{j}-q_{t r, i}-q_{v e, i}-\sum_{r \neq e} h_{r} \mathcal{J}_{r, i}
\end{array}\right)
$$

where the index $i$ refers to one of the three dimensions of space and $\delta$ is the Kronecker delta. $E$ is the total energy per unit volume while $E_{v e, m}$ and $e_{v e, m}$ represent respectively the vibrational energy per unit volume and the vibrational energy per unit mass for species $m . h_{s}$ is the enthalpy per unit mass of species $s$, while the pressure, $p$, is recovered from the partial pressures using Dalton's law. $\mathcal{J}_{s, i}$ are the components of the species mass diffusion vector and $\tau_{i j}$ represents the components of the viscous stress tensor.

The source term vector, $\dot{\boldsymbol{W}}$, is written as

$$
\dot{\boldsymbol{W}}=\left(0, \dot{\omega}_{s}, 0,0,0, \dot{\omega}_{v, m}, 0\right)^{T} \quad s \in N_{s}, m \in N_{m}
$$

where $\dot{\omega}_{s}$ is the net mass production of species $s$ and $\dot{\omega}_{v, m}$, for $m \in N_{m}$, is given by

$$
\dot{\omega}_{v, m}=Q_{m, V-T}+Q_{m, V-V}+Q_{m, C-V}
$$

In order of appearance in Equation 6, the source terms represent vibrational-translational (V-T) energy exchange, vibrational-vibrational (V-V) energy transfer and the vibrational-electronic energy added or removed by reactions to the species $m$. The default configuration assumes that the electronic mode is disabled. V-T energy exchange is modelled by the Landau-Teller equation [6]. The relaxation times are evaluated using either the Millikan and White [7] semi-empirical correlation with the correction of Park [8], and denoted as MWP in the following, or by the SSH theory named after Schwartz, Slawsky, and Herzfeld [9]. The coefficients of this latter model are taken from the work of Thivet [10] and a blended model is created with the MWP formulation for molecule-atom collisions. The formulation that has been implemented to account for V-V energy transfer is the one of Knab et al. [11, 12]. Finally, the vibration-chemistry source term is handled either by the Park TTV model [8] or by the coupled vibrationdissociation-vibration (CVDV) model of Marrone and Treanor [13, 14].

In hy2Foam, species thermal properties follow the Blottner [15] and Eucken [5, 16] formulas while mixture properties are recovered using the Armaly and Sutton mixing rule, as prescribed in the review from Palmer and Wright [17]. Other mixing rules such as those of Wilke [18] or Gupta [19] are also implemented but will not be considered in this work. The diffusion fluxes are governed by Fick's law with a correction term to ensure mass conservation [20]. The binary diffusion coefficient model is the one of Hirschfelder [21].

hy2Foam uses the central-upwind interpolation schemes of Kurganov and Tadmor [22]. The non-equilibrium boundary conditions employed at the wall are the first-order Smoluchowski temperature jump [23] and the Maxwell velocity slip [24].

\section{The direct simulation Monte Carlo method}

The direct simulation Monte Carlo (DSMC) method developed by Bird [25] is a particle-based methodology that is particularly well-suited for computing high Knudsen number flows, typically above 0.05. The DSMC code used in 
this work is the OpenFOAM solver $d s m c F o a m$ [26]. It inherently has three temperatures: translational, rotational and vibrational. The vibrational mode has been included in the latest release as a serial application of the quantum LarsenBorgnakke procedure. Thus, the vibrational energy can only take discrete quantum values and V-T energy exchanges rely mostly on microscopic gas information. Following on from this, the implementation of the quantum kinetic (QK) method to describe chemical reactions in a 5-species air model has been made possible.

\section{Departure from the continuum regime}

The local gradient-length Knudsen number initially proposed by Boyd [27] has extensively been used as a breakdown parameter in the literature (e.g. in $[28,29,30,31])$. This is computed to identify the regions where the continuum assumption does not hold. For a local macroscopic flow quantity $\phi$, it is defined as

$$
K n_{G L L-\phi}=\frac{\lambda}{\phi}|\nabla \phi|
$$

where $\lambda$ is the local mean free path of the gas molecules and in which $\phi$ can either be the gas density, the temperature, or the magnitude of the velocity vector $|\boldsymbol{U}|$ (or $\max (|\boldsymbol{U}|, a)$ in the denominator where $a$ is the speed of sound for low-speed regions [32]). The degree of local continuum breakdown is then evaluated as the maximum for all the aforementioned flow quantities

$$
K n_{G L L}=\max \left(K n_{G L L-\rho}, K n_{G L L-T_{t r}}, K n_{G L L-T_{v e}}, K n_{G L L-|U|}\right)
$$

This parameter will be informative in the subsequent simulations.

\section{RESULTS}

The hypersonic flow over a two-dimensional circular cylinder of radius $\mathrm{R}=1 \mathrm{~m}$ is being considered for analysis. The free-stream fluid is pure nitrogen. The geometry models the upper half of the domain thus taking advantage of the symmetry of the problem. The streamwise extent of the computational domain spans from $-1.8 \mathrm{~m}$ to $5 \mathrm{~m}$.

The initial conditions are listed in Table 1 . The case is run at a free-stream velocity of $6,047 \mathrm{~m} \mathrm{~s}^{-1}$ and pressure of $0.89 \mathrm{~Pa}$. The free-stream temperature $T_{\infty}=220 \mathrm{~K}$ is high enough to result in a vibrationally-excited flow-field. The cylinder wall is held at a uniform temperature of 1,000 K. The overall Knudsen number, equal to 0.0022, lies in the bottom range of the continuum-transition regime but locally the gas may lie into the transition regime.

TABLE 1. Initial conditions for the Mach 20 cylinder

\begin{tabular}{lll}
\hline \multicolumn{1}{c}{ Quantity } & \multicolumn{1}{c}{ Value } & \multicolumn{1}{c}{ Unit } \\
\hline Free-stream velocity, $U_{\infty}$ & 6,047 & $\mathrm{~m} \mathrm{~s}^{-1}$ \\
Free-stream pressure, $p_{\infty}$ & 0.89 & $\mathrm{~Pa}$ \\
Free-stream density, $\rho_{\infty}$ & $1.363 \times 10^{-5}$ & $\mathrm{~kg} \mathrm{~m}^{-3}$ \\
Free-stream temperature, $T_{\infty}$ & 220 & $\mathrm{~K}$ \\
Free-stream mean-free-path, $\lambda_{\infty}$ & $4.45 \times 10^{-3}$ & $\mathrm{~m}$ \\
Overall Knudsen number, $K n_{o v}$ & 0.0022 & - \\
Wall temperature, $T_{w}$ & 1,000 & $\mathrm{~K}$ \\
\hline
\end{tabular}

\section{CFD set-up}

The mesh used in this investigation consisted of 40,000 cells and the first spacing at the cylinder wall was taken as 2 microns. The simulations make the use of the Maxwell velocity slip and Smoluchowski temperature jump boundary conditions with accommodation coefficients equal to 1.

Both MWP and SSH formulations are successively used for V-T energy transfer for comparison purposes. The different set-ups are summarised in Table 2 together with a run identification number. 
TABLE 2. CFD simulations performed

\begin{tabular}{cccc}
\hline Run number & V-T transfer & Electronic mode & Rates \\
\hline 1 & MWP & no & - \\
2 & SSH & no & - \\
3 & MWP & yes & - \\
4 & MWP & no & QK \\
5 & SSH & no & QK \\
\hline
\end{tabular}

The two chemical reactions being considered in Run number 4 and 5 are the irreversible molecule-molecule and molecule-atom dissociation of nitrogen

$$
\begin{aligned}
& \mathrm{N}_{2}+\mathrm{N}_{2} \longrightarrow 2 \mathrm{~N}+\mathrm{N}_{2} \\
& \mathrm{~N}_{2}+\mathrm{N} \longrightarrow 2 \mathrm{~N}+\mathrm{N}^{2}
\end{aligned}
$$

The Arrhenius rate constants are shown in Table 3 in which the units of $A$ and $T_{a}$ are given in $\mathrm{m}^{3} \mathrm{~mol}^{-1} \mathrm{~s}^{-1}$ and Kelvin, respectively. They are derived from the QK theory [26]. Finally, the CVDV model is retained for each reacting computation.

TABLE 3. Parameters for the evaluation of the forward rate constant

\begin{tabular}{ccccc}
\hline \multirow{2}{*}{ Reaction rate } & Reaction & \multicolumn{3}{c}{ Arrhenius law constants } \\
& colliding partner & $\boldsymbol{A}$ & $\boldsymbol{\beta}$ & $\boldsymbol{T}_{\boldsymbol{a}}$ \\
\hline \multirow{2}{*}{$\mathrm{QK}$} & $\mathrm{N}_{2}$ & $2.47 \times 10^{18}$ & -0.62 & 113,500 \\
& $\mathrm{~N}$ & $6.02 \times 10^{18}$ & -0.68 & 113,500 \\
\hline
\end{tabular}

\section{DSMC set-up}

The variable hard sphere model is adopted with a temperature exponent of viscosity of 0.74 , and a reference temperature of $T_{r e f}=273 \mathrm{~K}$. The particles impinging the cylinder wall are reflected diffusely with an accommodation coefficient equal to 1 . Good DSMC practice have been satisfied for the mesh and the time-step. The cell size is equal to a third of the mean-free-path and a spline was used to minimize the region being modelled upstream the bow shock. The DSMC mesh then consisted of 5.5 million cells. Each cell was filled with approximately 15 equivalent DSMC particles which resulted in simulations using over 80 million particles. The DSMC time-step was set to $1 / 5$ of the mean-free-time.

\section{Analysis}

\section{Non-reacting case scenarios}

The regions where the flow departs significantly from local thermodynamic equilibrium are highlighted in Fig. 1(a) using the local gradient-length Knudsen number and later shown in Fig. 2. This indicates that the CFD solver will be unlikely to provide satisfactory results within the bow shock and in the near-wake of the cylinder, as shown by $K n_{G L L}$ values above 0.05 . In particular, the low density region for $\theta \simeq 130 \mathrm{deg}$ is driving the $K n_{G L L}$ beyond 10 . For the reacting case scenario, a very similar picture of $K n_{G L L}$ is observed.

As expected, the bow shock is more diffuse using the DSMC method as shown in Fig. 1(b). The downstream shock position is nearly identical using both solvers. The temperature fields in Fig. 1(c) and 1(d) are correctly approximated using hy2Foam for $x<0$, which corresponds to the region where the fluid undergoes compression. In the wake of the cylinder however, $T_{t r}$ is overall smaller and $T_{v}$ overall greater using CFD when compared with DSMC. These discrepancies show a lack of V-T energy redistribution in the expansion region, in which $T_{v}$ exceeds $T_{t r}$. Indeed, the quantum Larsen-Borgnakke method promotes a better energy harmonisation between the energy modes when $T_{t r}$ is low and $T_{v}$ is large when compared either with the SSH or with the Millikan-White semi-empirical correlation corrected by Park, models that are widespread in current CFD codes. 


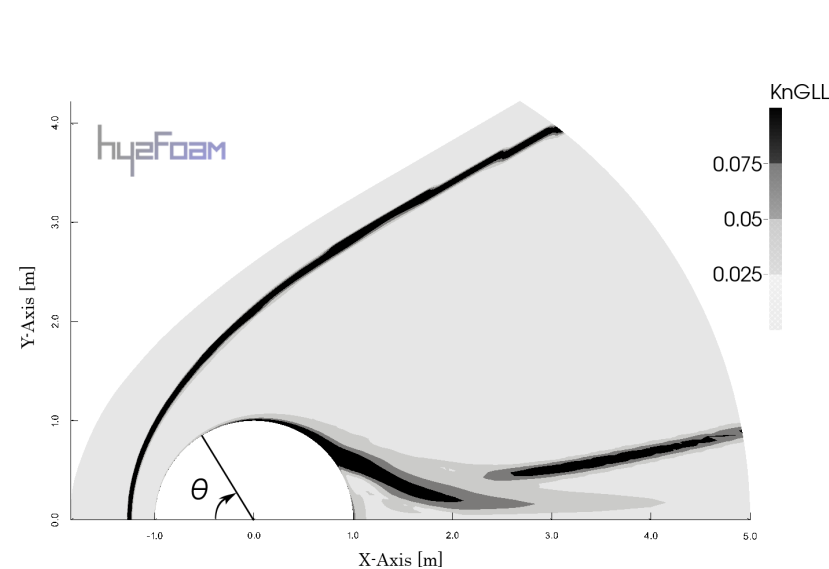

(a) Local gradient-length Knudsen number

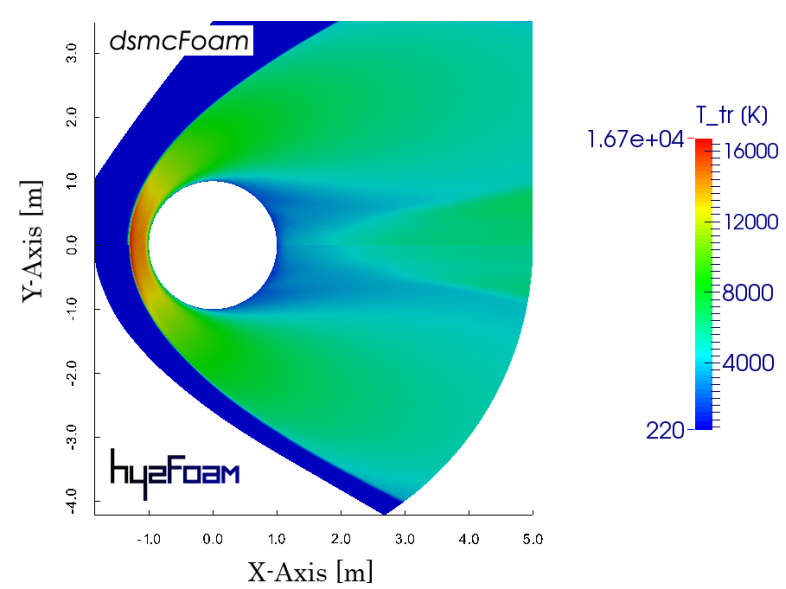

(c) Trans-rotational temperature

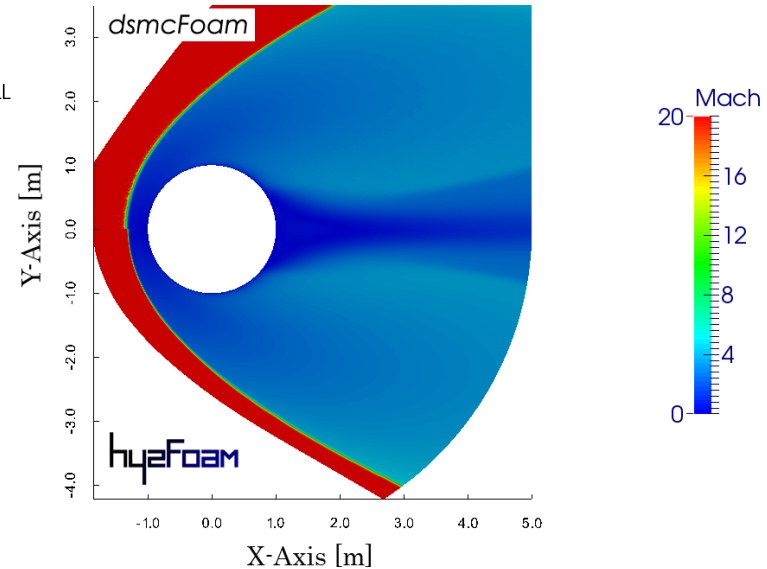

(b) Mach number

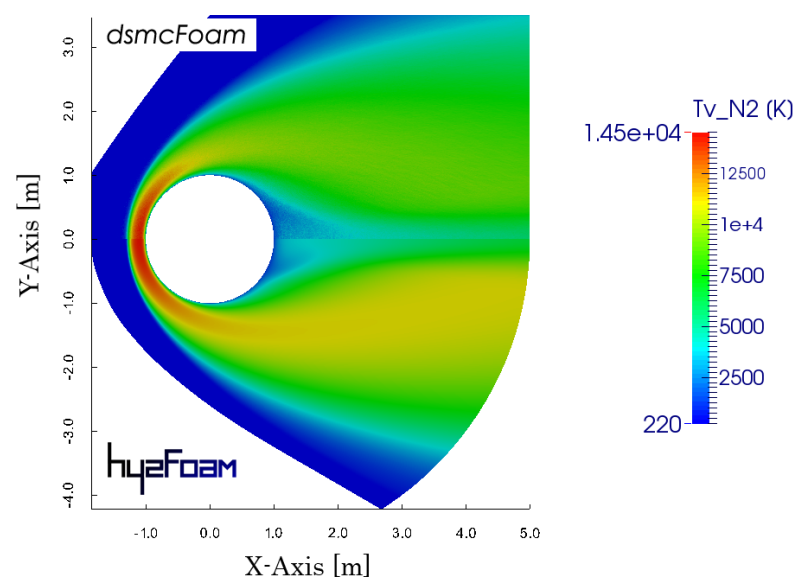

(d) Vibrational temperature

FIGURE 1. CFD-DSMC flow-field comparisons for Run number 2 (non-reacting).

Figure 2(a) - 2(c) compares the stagnation line profiles of temperature, number density, and Mach number provided by hy2Foam and $d s m c F o a m$ for the run number 2 . They are all in very good agreement outside of the $K n_{G L L}>0.1$ band.

\section{Effect of chemical reactions}

Figure 2(d) - 2(f) now include the effect of the two dissociation reactions considered. The shock stand-off distance is reduced from $0.29 \mathrm{~m}$ to $0.25 \mathrm{~m}$. Due to the significantly thin bow shock using CFD, the steep increase in $T_{t r}$ is delayed and the small production of atomic nitrogen within the shock is thus not captured, as seen in Fig. 2(d). The vibrational temperature profile is less accurately estimated as compared with the non-reacting case.

The surface properties for pressure coefficient, skin friction and heat transfer are shown in Fig. 3 for both nonreacting $(\mathrm{NR})$ and reacting $(\mathrm{QK})$ simulations. There is a reasonable agreement between the CFD and DSMC solvers.

Global aerothermodynamic coefficients for each simulation are given in Table 4. The drag coefficient estimated by hy2Foam presents less than $1.5 \%$ error and the reacting environment does not affect noticeably its value. The integrated heat flux however is nearly halved when considering the two dissociation reactions. The agreement remains satisfactory between the two solvers. Finally, the inclusion of the electronic mode in Run number 3 has shown to have very little effect on the global aerothermodynamic coefficients. 


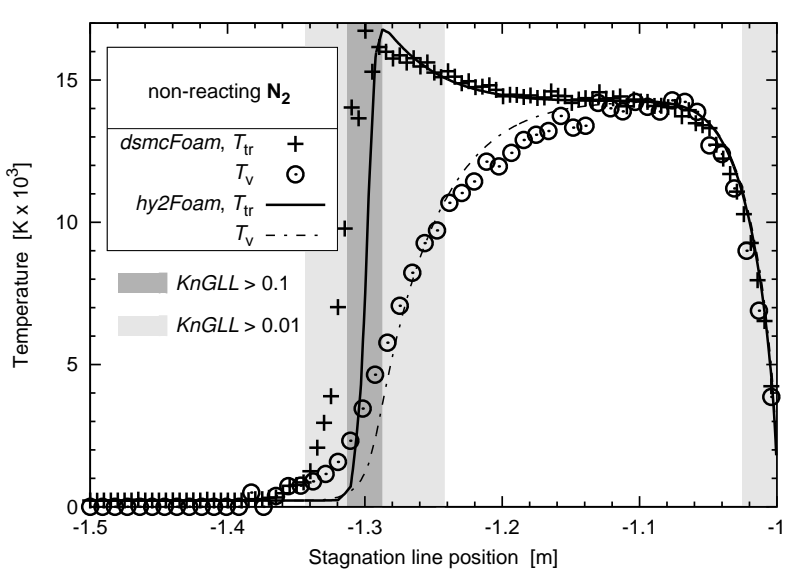

(a) Run 2: Temperature

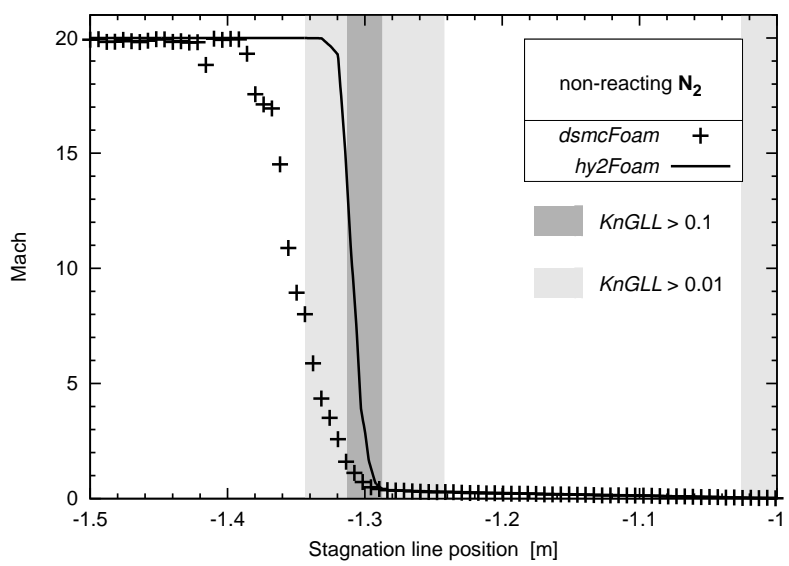

(c) Run 2: Mach number

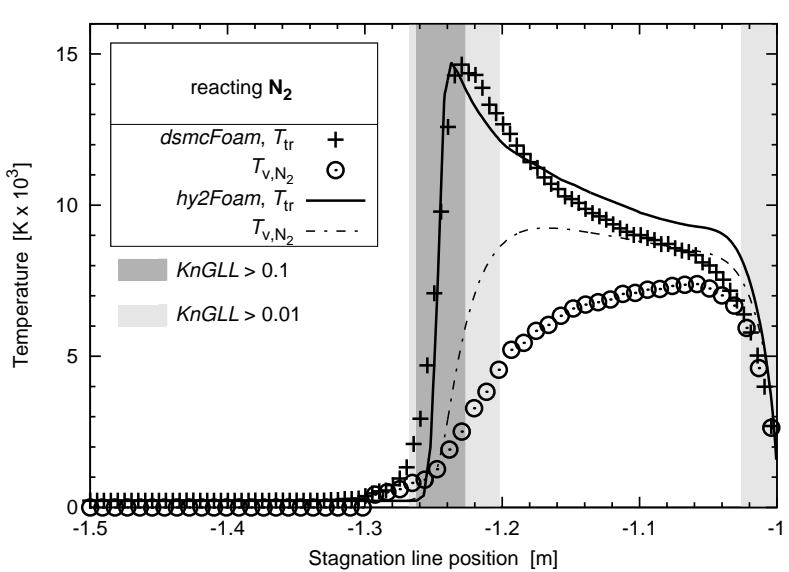

(e) Run 5: Temperature

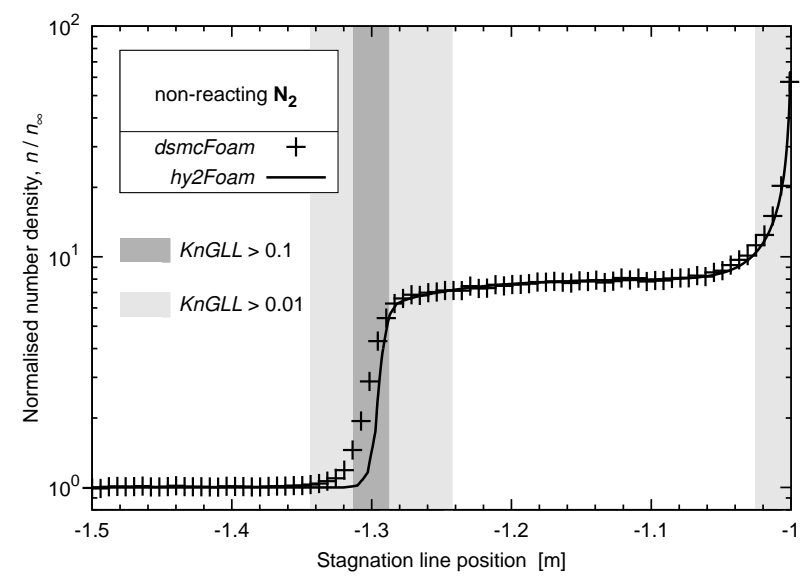

(b) Run 2: Normalised number density

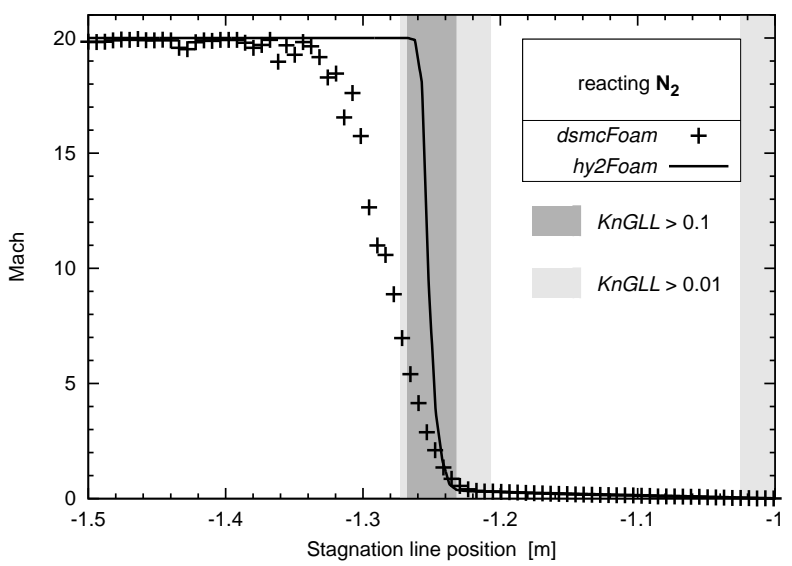

(d) Run 5: Mach number

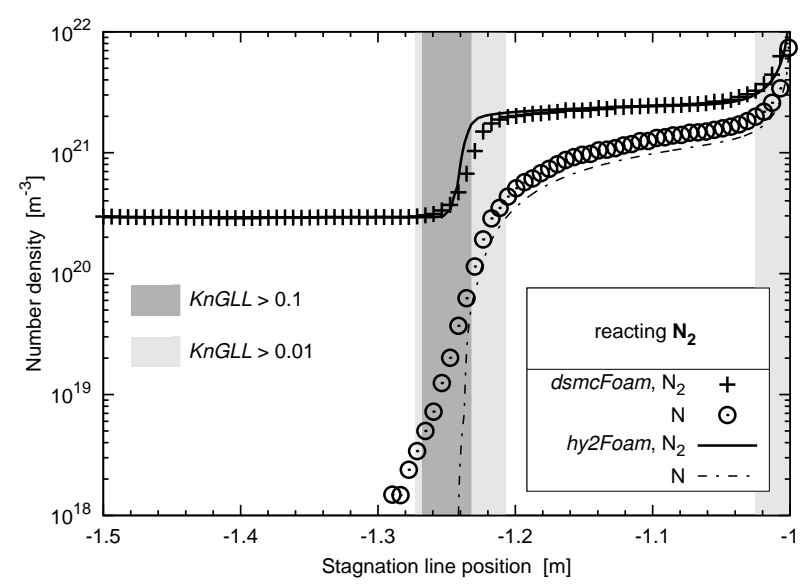

(f) Run 5: Number density

FIGURE 2. Stagnation line profiles for the run numbers 2 and 5. 


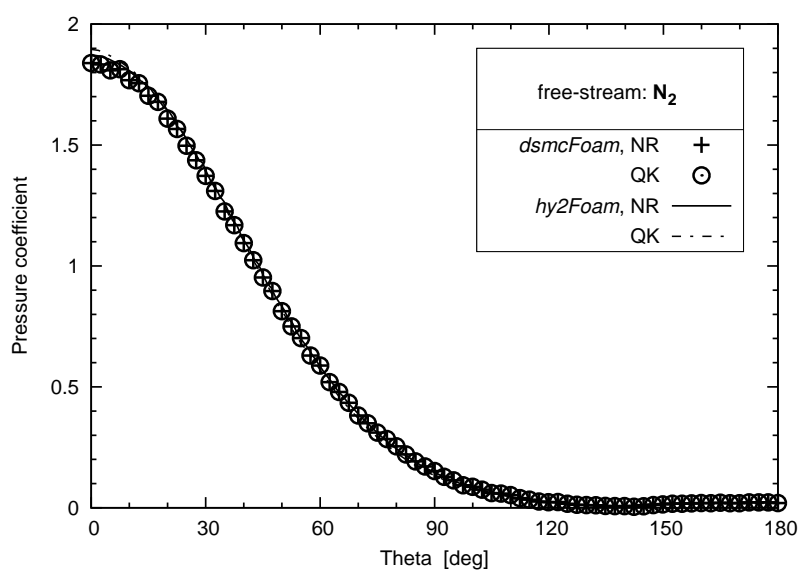

(a) Pressure coefficient

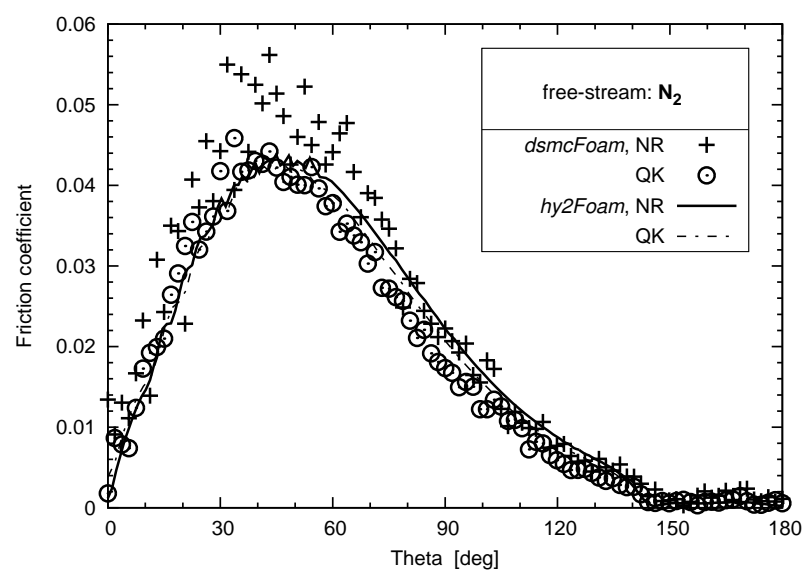

(b) Skin-friction coefficient

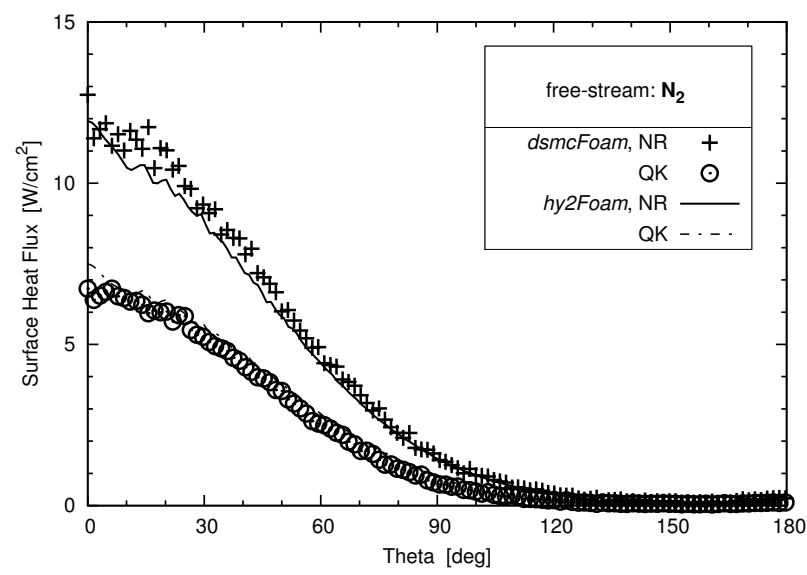

(c) Surface heat transfer

FIGURE 3. Surface quantities around the cylinder (run numbers 2 and 5).

TABLE 4. Global aerothermodynamic coefficients

\begin{tabular}{ccccc} 
CFD run number & \multicolumn{2}{c}{$\boldsymbol{C}_{\boldsymbol{D}}$ (pressure contribution) } & \multicolumn{2}{c}{$\boldsymbol{C}_{\boldsymbol{H}}[\mathbf{k W}]$} \\
& $\mathbf{C F D}$ & DSMC & CFD & DSMC \\
\hline 1 & $1.3(96.8 \%)$ & $1.286(97.5 \%)$ & 115 & 115 \\
2 & $1.296(96.8 \%)$ & $\times$ & 114 & $\times$ \\
3 & $1.308(96.8 \%)$ & $\times$ & 66.5 & 63.3 \\
4 & $1.3(96.8 \%)$ & $1.284(97.8 \%)$ & 68.2 &
\end{tabular}

\section{CONCLUSIONS}

The hy2Foam and $d s m c F o a m$ results have shown to be in reasonable concordance, thus verifying the implementation of hy2Foam for two-dimensional geometries in a reacting and neutral environment. The use of the CVDV model for chemistry-vibration coupling together with reaction rates derived from Quantum-Kinetics -through the use of $d s m c F o a m$ - has shown to be an appropriate choice. Hence, it is thought that the verification case scenario presented in this paper provides a useful basis for other codes to compare against. Future work will focus on simulating this same geometry at higher Knudsen numbers using air and thus including more chemical reactions. 


\section{ACKNOWLEDGMENTS}

The CFD results were obtained using the EPSRC funded ARCHIE-WeSt High Performance Computer (www.archiewest.ac.uk/). EPSRC grant no. EP/K000586/1. This work has also been awarded an ARCHER Resource Allocation Panel (RAP) grant. D. R. Emerson and B. John thank EPSRC for support under grants EP/N016602/1 and EP/K038427/1. V. Casseau would like to acknowledge Daniel E. R. Espinoza and Jimmy-John Hoste for their help in testing the hy2Foam solver.

\section{REFERENCES}

[1] T. Schwartzentruber and I. D. Boyd, Progress in Aerospace Sciences 72, 66-79 (2014).

[2] D. E. R. Espinoza, V. Casseau, T. J. Scanlon, and R. E. Brown, "An Open Source Hybrid CFD-DSMC Solver for High-Speed Flows," in Proceedings of the 30th Rarefied Gas Dynamics Conference, Paper 2964 (Victoria BC, Canada, July 10-15, 2016).

[3] V. Casseau, T. J. Scanlon, and R. E. Brown, "Development of a Two-Temperature Open-Source CFD Model for Hypersonic Reacting Flows," AIAA Paper 2015-3637 (2015).

[4] OpenFOAM official website, http://www.openfoam.com/ ( accessed: 12 January 2016),

[5] G. V. Candler and I. Nompelis, "Computational Fluid Dynamics for Atmospheric Entry," RTO-EN-AVT-162 (2009).

[6] L. Landau and E. Teller, Physikalische Zeitschrift der Sowjetunion 10 (1936).

[7] R. C. Millikan and D. R. White, The Journal of Chemical Physics 39, 3209-3213 (1963).

[8] C. Park, Nonequilibrium Hypersonic Aerothermodynamics (Wiley International, New York, 1990).

[9] R. N. Schwartz, Z. I. Slawsky, and K. F. Herzfeld, The Journal of Chemical Physics 20, 1591-1599 (1952).

[10] F. Thivet, M. Y. Perrin, and S. Candel, Physics of Fluids 3, 2799-2812 (1991).

[11] O. Knab, H.-H. Frühauf, and S. Jonas, "Multiple Temperature Descriptions of Reaction Rate Constants with Regard to Consistent Chemical-Vibrational Coupling," AIAA Paper 92-2947 (1992).

[12] O. Knab, H.-H. Frühauf, and E. W. Messerschmid, Journal of Thermophysics and Heat Transfer 9, 219-226 (1995).

[13] C. E. Treanor and P. V. Marrone, Physics of Fluids 9, 1022-1026 (1962).

[14] P. V. Marrone and C. E. Treanor, Physics of Fluids 6, 1215-1221 (1963).

[15] F. G. Blottner, M. Johnson, and M. Ellis, "Chemically reacting viscous flow program for multicomponent gas mixtures," Report No. SC-RR-70-754 (Albuquerque, New Mexico, 1971).

[16] W. G. Vincenti and C. H. Kruger, Introduction to Physical Gas Dynamics (Krieger Publishing Company, Florida, 1965).

[17] G. E. Palmer and M. J. Wright, Journal of Thermophysics and Heat Transfer 17, 232-239 (2003).

[18] C. R. Wilke, Journal of Chemical Physics 18, 517-519 (1950).

[19] R. Gupta, J. M. Yos, R. A. Thompson, and K.-P. Lee, "A Review of Reaction Rates and Thermodynamic and Transport Properties for an 11-Species Air Model for Chemical and Thermal Nonequilbrium Calculations to 30000 K," Tech. Rep.

[20] K. Sutton and P. A. Gnoffo, "Multi-component Diffusion with Application to Computational Aerothermodynamics," AIAA Paper 98-2575 (1998).

[21] J. O. Hirschfelder, C. Curtiss, and R. B. Bird, Molecular Theory of Gases and Liquids (John Wiley \& Sons, Inc., 1954).

[22] A. Kurganov, S. Noelle, and G. Petrova, SIAM J. Sci. Comput. 23, 707-740 (2001).

[23] M. von Smoluchowski, Annalen der Physik und Chemie 64, 101-130 (1898).

[24] J. C. Maxwell, Phil. Trans. R. Soc. Lond. 170, 231-256 (1879).

[25] G. A. Bird, Molecular gas dynamics and the direct simulation of gas flows (Clarendon, Oxford, 1994).

[26] T. J. Scanlon et al., AIAA Journal 53, 1670-1680 (2015).

[27] I. D. Boyd, G. Chen, and G. V. Candler, Physics of Fluids 7, 210-219 (1995).

[28] T. E. Schwartzentruber, L. C. Scalabrin, and I. D. Boyd, J. Comput. Phys. 225, 1159-1174 (2007).

[29] G. Abbate, B. J. Thijsse, and C. R. Kleijn, "Computational Science - ICCS 2007: 7th International Conference, Beijing, China, May 27 - 30, 2007, Proceedings, Part I,” (Springer Berlin Heidelberg, Berlin, Heidelberg, 2007) Chap. Coupled Navier-Stokes/DSMC Method for Transient and Steady-State Gas Flows,, pp. 842-849.

[30] M. Darbandi and E. Roohi, Sensors and Actuators A 189, 409-419 (2013).

[31] R. Roveda, D. B. Goldstein, and P. L. Varghese, J. Spacecraft Rockets 35, 258-265 (1998).

[32] T. E. Schwartzentruber, L. C. Scalabrin, and I. D. Boyd, Journal of Thermodynamics and Heat Transfer 22, 29-37 (2008). 